\title{
Computer-Assisted Orthognathic Surgery: Consequences of a Clinical Evaluation
}

\author{
G. Bettega, $\mathrm{MD}, \mathrm{PhD}^{1}$, F.Leitner, $\mathrm{PhD}^{2}$, O. Raoult, $\mathrm{PhD}^{3}$, V. Dessenne, $\mathrm{PhD}^{4}$, \\ P. Cinquin, $\mathrm{PhD}^{5}$, B. Raphael, $\mathrm{MD}, \mathrm{PhD}^{1}$
}

1 Plastic and Maxillo-Facial Surgery Department, CHU. A. Michallon, BP 217. 38043 GRENOBLE Cedex 09. FRANCE.

E-mail: GBettega@,chu-grenoble.fr

2 AESCULAP AG, Am Aesculap Platz. Postfach 40, 78000 Tuttlingen. GERMANY.

310 chemin du Clapero, 38360 Sassenage. FRANCE.

MEDIVISION, Müller Institut für Biomechanik, Durtenstrasse 35, Postfach 30, CH-3010

Bern, SWITZERLAND

5 TIMC-IMAG, institut A. Bonniot, Faculté de Médecine de Grenoble, 38700 La Tronche, FRANCE.

\begin{abstract}
Purpose and method: Computer-assisted systems, as any medical technology, have to go through several steps within an endless loop before becoming routine. The first step is the conception of a prototype, the second is its adaptation to the clinical modalities (via animals or cadavers studies), the third is the clinical validation and the last one is the ergonomics optimization preceding the industrialization.

The aim of this work is to present the evolution of a computer-assisted system built for orthognathic surgery i.e. for the surgery of the maxilla and more specifically for repositioning the mandibular condyle after sagittal split osteotomies. The system was based on three-dimensional optical localization of infrared emitting diodes. Eleven patients ("empirical group") underwent condylar repositioning using the empirical repositioning method (standard technique) and were considered controls. In ten patients ("active group") the computer-assisted system was used to replace the condyle bearing fragment in its sagittal preoperative position ; in these cases the condylar torque wasn't controlled. In the third group of ten patients ("graft group"), the computer-assisted system was used to replace the condyle in all three directions ; very often it was necessary, in this group, to fill the osteotomy gap by a bone graft. The clinical evaluation was based on four major criteria: the quality of postoperative dental occlusion, the stability of skeletal position on successive teleradiographies, the occurrence of temporo-mandibular dysfunction (TMJD), and the preservation of mandibular motion. Clinical assessment was made at 1, 3, 6 and 12 months of follow-up. Results: Forty-five percent of the "empirical group" patients do not have the expected postoperative occlusion, five patients showed evidence of clinical relapse at one year, forty-five percent worsened their TMJD status, and they recovered only $63.37 \%$ of their mandibular motion amplitudes at 6 months. All the patients of the "active group" had the expected occlusion, only one patient exhibited a mild relapse and TMJD symptoms, and the average mandibular
\end{abstract}


motion recovery was only $62.65 \%$ at 6 months. All the patients of the "graft group" had a good occlusion and no relapse or TMJD. Their percentage of mandibular motion recovery was $77.58 \%$.

Conclusion: Those results confirm the utility of a condyle repositioning system. They also prove the accuracy of this computer-assisted method. This lead to an improvement of the system using a smaller localizer and a simple PC directly commanded by the surgeon with a foot-switch, without any technical support. The surgical ancillary is also simplified to reduce the time needed for the setting up of the sensors. The simplified system became a CE marked product and it is now routinely used for every orthognathic procedure in our department.

Key words: Clinical results - Surgical navigation systems - Orthognathic surgery -

\section{Introduction}

Computer-assisted systems, as any medical technology, have to go through several steps before becoming routine. The first step is the conception of a prototype, the second is its adaptation to the clinical modalities (via animals or cadavers studies), the third is the clinical validation and the last one is the ergonomic optimization preceding the industrialization. The aim of this work is to present the evolution of a computer-assisted system built for orthognathic surgery.

Orthognathic surgery aims to correct dentofacial deformities. The operation have been greatly improved thanks to major advances in anesthesiology and to the improvement of osteosynthesis materials. Nevertheless, many problems are still encountered, creating sources for inaccuracy and instability. Amongst these difficulties, one predominates: positioning of the condyle (the articular fragment) after osteotomy of the mandible. The quality of the procedure is closely related to the operator's experience. Joint laxity and muscular relaxation due to general anesthesia, are two factors which complicate this surgical maneuver. EPKER ${ }^{1}$ suggests 3 main reasons to justify a precise repositioning of the condyle fragment during sagittal split osteotomies of the mandibule ${ }^{1}$. The first, and the main reason, is to ensure long-term stability of the surgical result. The second is to reduce noxious effects on the temporomandibular joint and notably to reduce the incidence of temporomandibular joint dysfunction (TMJD). Finally, it may improve postoperative masticatory function. VAN SICKEL ${ }^{2}$ confirmed this in a more recent article.

The empirical method is the most widely used method to reposition the condyle fragment after a mandibular osteotomy. It consists in trying to place manually the condyle in its most superior and anterior position within the glenoid cavity ${ }^{3}$. As simple as it is, this method is quite dependent on the surgeon's experience. This is why many mechanical systems have been described. Today, none are really used in common practice and it is difficult to quantify the advantages brought by these repositioning systems since none have really been clinically evaluated. In 1996, we described an original computer-assisted procedure based on the three-dimensional localization optical principle of an infrared transmitter ${ }^{4}$.

We present the clinical evaluation of this prototype. These results lead us to improve the system in a way to obtain a routinely used CE marked product. These important evolutions are presented. 


\section{The Clinical Validation}

\subsection{Material and Method}

The system consists of a 3D optical localizer (Optotrak ${ }^{\mathrm{TM}}$ Northern Digital) and a workstation (DEC Alpha, OSF/1) installed on a gurney adapted to dimensions of the operating room. The optical localizer tracks 4 infrared emitting diodes assembled on a small silicon plate called "rigid body". These rigid bodies feature an attachment component making it possible to anchor them to osseous parts. Four rigid bodies are used in this application (figure 1). One is assembled on a probe, which makes it possible to define a reference mark on the patient, the 3 others are anchored on an osseous part: one on a structure not moved by the osteotomies, and used as reference (the selected site was arbitrarily the left upper orbital ridge); the two others on each condyle. Fixation to the orbit is inserted with Kirchner pins transcutaneously; an intermediary part is secured to these pins; the corresponding rigid-body can be attached or removed from this part as often as needed. Condyle anchoring is performed via a mini-plate secured with monocortical screws at the mandibular angle (figure 2); this plate is equipped with a connection which can hold a removable intermediary part, supporting the corresponding rigid-body. This plate is placed transorally using transbuccal instrumentation. Anchoring with $35-\mathrm{mm}$ screws provides extreme stability and does not interfere with the sagittal split osteotomy. Surgery thus begins by installation of rigid bodies(figure 3 ). The next step consists in recording the two condyles' reference position using an intercuspidation splint established during preoperative assessment. The optical localizer determines the position of each condyle's rigid body in relation to the rigid body on the eyebrow. The rigid bodies are then removed and only the osseous anchorage remains, which completely frees the operative field. The surgeon can then perform the mandibular osteotomy of his/her choice without modifying the traditional technique. Maxillomandibular fixation is then secured and the rigid bodies are reinserted on their respective anchorage so that the surgical navigation stage may begin. The latter consists of tracking condylar displacements on computer screen while trying to make its representation overlap the reference position.

The operated patients were randomized by drawing into 3 groups. The first group named "empirical" was all the patients for whom the most experienced surgeon performed a condyle repositioning using the traditional technique, choosing the most superior and anterior position in the glenoid fossa. Computer assistance was only used at the end of surgery to measure possible displacement in relation to the reference position. In the second group named "active", the condyle was repositioned with computer assistance but only in the sagittal plane. The third group named "grafted" corresponds to all the patients whose condyles were repositioned with computer assistance but this time, the position was controlled in three dimensions. In this group, for a significant number of cases, it was necessary to maintain a gap between the various osteotomized osseous fragments by using an osseous graft or an osseous substitute in order to avoid axial or frontal displacement. Four criteria: 2 anatomical and 2 functional were used to assess outcomes. The first anatomical criterion was the evaluation of dental occlusion by comparing post osteotomy occlusion with the planned occlusion. The result is binary i.e. it is regarded as good if the patient perfectly sets in the final intercuspidation plate; it is regarded as bad if it does not. The 
second anatomical criterion was the stability of mandibular structures on successive cephalometric radiographs. It was studied by superposing the late postoperative cephalograms over the immediate postoperative cephalogram (before the eighth day). The degree of displacement or relapse was measured by comparing the displacement of various points along the symphysis from one cephalogram to the next. The result was regarded as good if there was no displacement, it was regarded as bad if displacement was greater than $2 \mathrm{~mm}$, between 0 and $2 \mathrm{~mm}$ the result was labeled as dubious due to possible superposition errors. The first functional evaluation criterion was a possible temporo-mandibular joint dysfunction (TMJD). Temporomandibular joint (TMJ) related symptoms were compared before and after surgery. The results of TMJD assessment was regarded as good if the preoperative status was preserved or improved, otherwise it was regarded as bad. The last evaluation criterion was that of masticatory function assessed with a slide caliper by measuring the amplitudes of movement in opening, in protrusion, and lateral movement. The result was expressed as a percentage of preoperative amplitude. All these criteria were evaluated 1 month, 3 months, 6 months, and a year after surgery. All the patients were operated by the same surgeon and received the same postoperative care.

\subsection{Results}

On the whole, 31 patients were evaluated: 11 patients were included in the "empirical" group, 10 patients in the "active" group, and 10 patients in the "grafted" group. No specific complication related to the use of the system was noted.

\begin{tabular}{|c|c|c|c|c|c|c|c|c|c|c|c|c|c|c|c|c|c|c|}
\hline & \multicolumn{3}{|c|}{$\begin{array}{c}\text { Right-left } \\
\text { translation (mm) }\end{array}$} & \multicolumn{3}{|c|}{$\begin{array}{c}\text { Up-down } \\
\text { translation (mm) }\end{array}$} & \multicolumn{3}{|c|}{$\begin{array}{c}\text { Back-front } \\
\text { translation }(\mathrm{mm})\end{array}$} & \multicolumn{3}{|c|}{$\begin{array}{l}\text { frontal rotation } \\
\text { (degrees) }\end{array}$} & \multicolumn{3}{|c|}{$\begin{array}{l}\text { axial rotation } \\
\text { (degrees) }\end{array}$} & \multicolumn{3}{|c|}{$\begin{array}{c}\text { sagittal } \\
\text { rotation (de- } \\
\text { grees) }\end{array}$} \\
\hline & $\begin{array}{l}\frac{0}{3} \\
\frac{3}{\bar{y}} \\
\underline{\bar{D}}\end{array}$ & 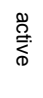 & 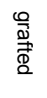 & 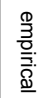 & 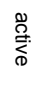 & 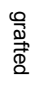 & $\begin{array}{l}\stackrel{\mathbb{1}}{3} \\
\text { 흘: } \\
\underline{\overline{0}}\end{array}$ & 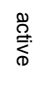 & 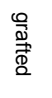 & $\begin{array}{l}\frac{10}{3} \\
\frac{3}{\bar{B}} \\
\underline{0}\end{array}$ & 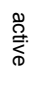 & $\begin{array}{l}\frac{0}{3} \\
\frac{0}{\mathbb{D}} \\
\frac{D}{2}\end{array}$ & $\begin{array}{l}\frac{10}{3} \\
\frac{3}{\bar{B}} \\
\underline{0}\end{array}$ & 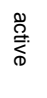 & 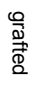 & $\begin{array}{l}\frac{1}{3} \\
\frac{3}{\bar{D}} \\
\underline{\bar{D}}\end{array}$ & 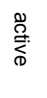 & $\begin{array}{l}\stackrel{0}{0} \\
\stackrel{\mathbb{D}}{\vec{D}} \\
\stackrel{D}{Q}\end{array}$ \\
\hline mean & 2,2 & 0,9 & 0,6 & 2,0 & 1,1 & 1,2 & 1,4 & 1,6 & 1,1 & 4,0 & 3,6 & 1,3 & 3,2 & 2,5 & 2,0 & 3,9 & 1,1 & 0,8 \\
\hline $\begin{array}{l}\text { Stan- } \\
\text { dard } \\
\text { devia- } \\
\text { tion }\end{array}$ & 1,8 & 0,6 & 0,4 & 1,4 & 1,1 & 0,6 & 1,0 & 1,2 & 0,9 & 2,4 & 2,3 & 1,0 & 2,2 & 2,1 & 1,0 & 3,4 & 1,2 & 0,8 \\
\hline $\begin{array}{l}\text { mini- } \\
\text { mum }\end{array}$ & 0,1 & 0,0 & 0,0 & 0,1 & 0,1 & 0,0 & 0,2 & 0,0 & 0,1 & 0,1 & 0,4 & 0,0 & 0,0 & 0,1 & 0,5 & 0,0 & 0,0 & 0,1 \\
\hline $\begin{array}{l}\operatorname{maxi}- \\
\text { mum }\end{array}$ & 6,6 & 1,9 & 1,5 & 4,7 & 4,0 & 2,4 & 3,6 & 4,0 & 3,5 & 7,5 & 7,4 & 4,2 & 8,4 & 9,8 & 3,5 & 11,3 & 4,3 & 1,7 \\
\hline
\end{tabular}

Table 1: values of displacement of the condyle measured by the computer-assisted system

Table 1 summarized the mean values, standard deviation, minimum and maximum displacements of the condyle, measured by the system. The mean displacements were greater, in all directions, for the "empirical" group and the dispersion of the values was also more important. Conversely, the mean values of displacement in the "grafted" group turned around one degree or one millimeter. The standard deviation and the maximum of displacement were also lower for the "grafted" patients than in 
the other groups, showing a better reproducibility of repositioning. But the maximum rotation values in the frontal and axial plans (respectively 4,2 degrees and 3,5 degrees) attested the difficulties to stabilize, in these directions the condylar bearing fragment during the osteosynthesis. Nevertheless, the repositioning is still better than in the "empirical" and the "active" group. The control of the front/back translation was similar in each group.

Postoperative dental occlusion analysis showed that approximately $45 \%$ of the "empirical" patients did not have the anticipated occlusal result (figure 4). Indeed 5 of the 11 patients required active orthodontics to correct malocclusion, although in most cases, they were not very significant. Nevertheless, only 3 of them, after 6 months of treatment, had obtained a satisfactory occlusion. All the patients of the "active" group and of the "grafted" group presented an occlusion strictly in conformity with that planned. None of them needed postoperative orthodontics. No patients in the "grafted" group presented any relapse at one year of follow-up (figure 5). One patient in the "active" group had a relatively minor relapse (about $1 \mathrm{~mm}$ ). On the other hand, in the "empirical" group, 3 patients had a relatively minor relapse, less than $2 \mathrm{~mm}$, and 2 other patients showed more than $2 \mathrm{~mm}$ relapse and required a second surgery. In the first case, 1 year after the first surgery because of a class III relapse, in the second case, 2 days after.

From a functional point of view, the results were comparable insofar as in the "empirical" group 5 patients had a TMJD or worsened a preexistent TMJD, even though none of them needed a particular treatment (figure 6). In the "active" group, only 1 patient worsened a preexistent TMJD whereas all the patients of the "grafted" group kept same the symptomatology of their significant difference between "grafted" patients and the other patients was noted at the sixth postoperative month follow-up, in terms of mandibular motion amplitude recovery (figure 7). Indeed, the average recovery percentage was the same for the "empirical" and "activē" groups (respectively $63.37 \%$ and $62.65 \%$ ) with a very significant variance in both groups. On the contrary, for the "grafted" group, not only the percentage of recovery was much higher $(77.58 \%)$ but the variance was also much less, demonstrating better reproductivity of the results.

Using computer assistance lead to lengthening the duration of surgery by approximately 50 minutes. This time increase is partly due to the setting up of various elements of the system (approximately $50 \%$ of the time increase). This excludes the extra time necessary for the adaptation of the bone fragments and the graft after repositioning, in order to allow for a stable synthesis.

The scar left after insertion of the eyebrow reference was not noticeable.

\subsection{Discussion}

Our study featured an objective and quantified comparison between a control population (the "empirical" group) and patients treated with computer assistance. The "active" group was created to check whether the sole control of sagittal condyle repositioning was sufficient to guarantee a satisfactory clinical result. Our results confirm the worth of a condyle repositioning system, especially for anatomical results since 
postoperative occlusal disorders and relapse was much more significant in the "empirical" group. The comparison with the "active" group and the "grafted" group showed that the precision of sagittal repositioning was the principal factor in the quality of postoperative occlusion and skeletal stability. On the other hand, the functional result was correlated to axial and frontal rotation control, especially for the recovery of mandibular motion amplitude. Moreover, the current prototype version of the system lengthens surgery by approximately 50 minutes. Half of this lengthening is due to the setting up in of the various sensors. Another advantage of this computerassisted system is that the interface gives the surgeon quantitative and spatial data compared to the chosen reference position. If the surgeon estimates that it is not desirable to reproduce the planned position exactly, he can vary repositioning in the direction of his choice and with the amplitude that he considers adapted.

The precision of this method reaches $\pm 0.5 \mathrm{~mm}$ and $\pm 0.5^{\circ}$. But this level of performance is one which the surgeon's hand cannot reach. In our experiment, it seemed almost impossible to reach a surgical precision below 1 millimeter, taking into account the irregularity of osseous cut and surgical constraints. In any case, clinical results show that in practice this precision is largely sufficient.

Those results confirm the utility of a condyle repositioning system. They also prove the accuracy of this computer-assisted method. But with this first version of the system the surgeon needed the help of a technician to deal with the computer. Moreover the lengthening of the procedure was incompatible with a routine application. To reach this objective it was necessary to improve and simplify the system.

\section{Evolution of the Prototype}

\subsection{Evolution of the Navigation System}

The initial system was somehow cumbersome and quite difficult to move (Figure 8A). A Unix workstation was necessary to run the application and a PC was dedicated to localizer management. Another disadvantage, for the surgeon, was his dependence to a technician during the procedure. So the system was drastically modify.

The localizer was changed for a smaller one (Polaris ${ }^{\mathrm{TM}}$ Northern Digital). Even with less accuracy, it was sufficient for our application to take into account the accuracy of the surgical procedure. All software (localizer management, and application) was concentrated into a simple PC (even a laptop) directly controlled by the surgeon through a double foot-switch (Figure 8B).

The new platform is very easy to install, it can be integrated into the operating room and managed by nurses as a classical maxillo-facial instrumentation. The great mobility of the system permits to share it with other teams.

\subsection{Evolution of the Ancillary}

The other ergonomic problem was the lengthening of the procedure, due essentially to fixation of condyle anchoring. The small plate was very satisfactory in a mechanical point of view. It was also very stable and it did not perturb the realization of the osteotomy. However it needed around 10 minutes installation per side. Moreover it was 
necessary to take off the rigid bodies during the mandibular screw fixation; so that it was impossible to control the condyle position during the osteosynthesis. This control had to be done later after re-installation of the rigid bodies. All this was time consuming (around 10 to 15 more minutes per side ).

Then we replaced these plates by small endo-orally fixed vises, on the coronoid process of the mandible (Figure 9). It is simply done using a specific ancillary in less than 1 minute per side. The transbuccal approach is left free so it is possible to control condyle position during osteosynthesis with the rigid bodies in place. The total gain in time varies from 40 to 45 minutes

With this new ancillary the mean lengthening of the intervention is 10 to 15 minutes (mostly due to the insertion of the reference rigid body on the eyebrow).

The new system (Orthopilot ${ }^{\mathrm{TM}}$, BBraun Aesculap) is now adapted to routine application in orthognathic surgery. It is now used systematically in our center for this type of surgery.

\section{References:}

1- Epker BN, Wylie GA.- Control of the condylar-proximal mandibular segments after sagittal split osteotomies to advance the mandibule. Oral Surg Oral Med Oral Pathol,62:613-7, 1986. 2- Van Sickel JE, Don Tiner B, Alder ME: Condylar torque as a possible cause of hypomobility after sagittal split osteotomy: report of three cases. J Oral Maxillofac Surg 55:398-402,1997. 3- Bell WH, Profitt WR, White RP: Surgical correction of dentofacial deformities, volume 2. Philadelphia, PA, Saunders, 1980,p910

4- Bettega G, Dessenne V, Cinquin P, Raphaël B. Computer assisted mandibular condyle positioning in orthognathic surgery. J. Oral Maxillofac. Surg. 54(5):553-8, 1996. 


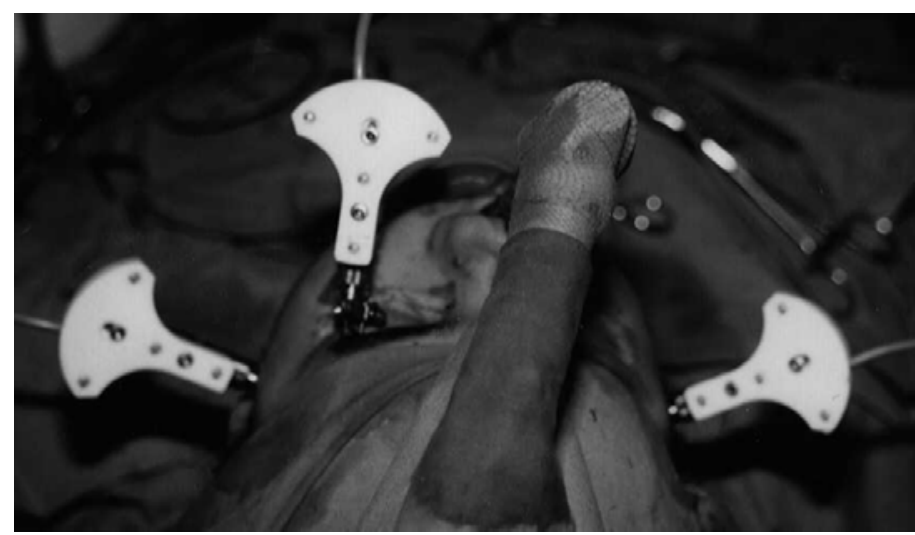

Figure 1: The fixation of the rigid-bodies on the patient. The reference rigid body is place on the left upper orbital ridge (which is not concerned by the osteotomy) and the others are place on each condylar fragment.

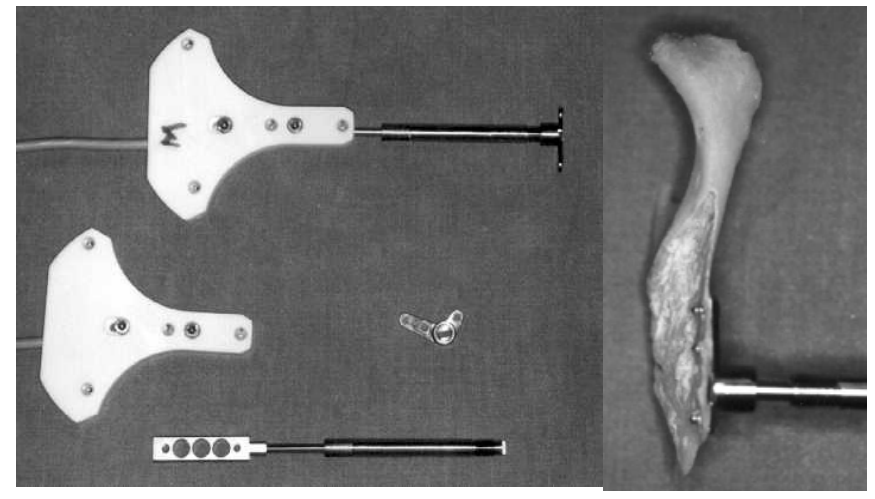

Figure 2: the condyle anchoring system. It included:

- a mini-plate fixed by three screws on the external aspect of the mandibular angle,

- $\quad$ a rod on which is secured the rigid body.

The connection between the rod and the plate is reversible. 


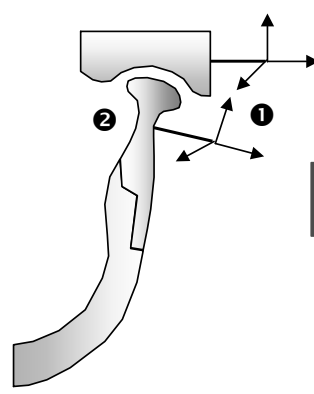

D Installing the rigidbodies

2 recording of initial condyle position (centric relation)

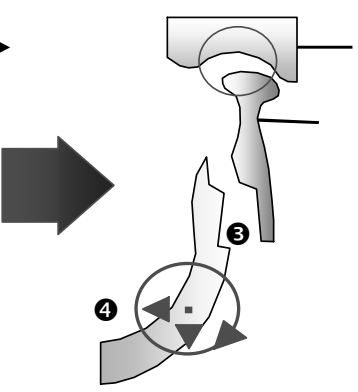

3 Mandibular osteotomy 4 mobilization and fixation of the dental segment taking into account the occlusion

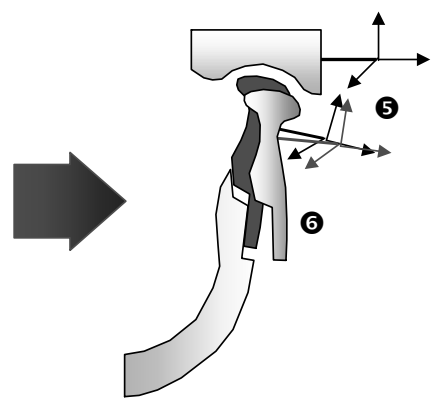

5 Re-installation of the rigid-bodies

6 navigation

Figure 3: the algorithm of the surgical procedure

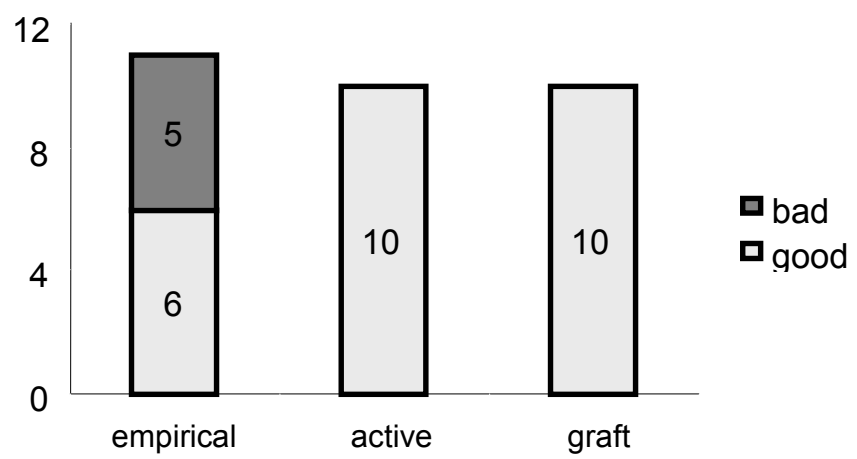

Figure 4: Quality of postoperative occlusion 


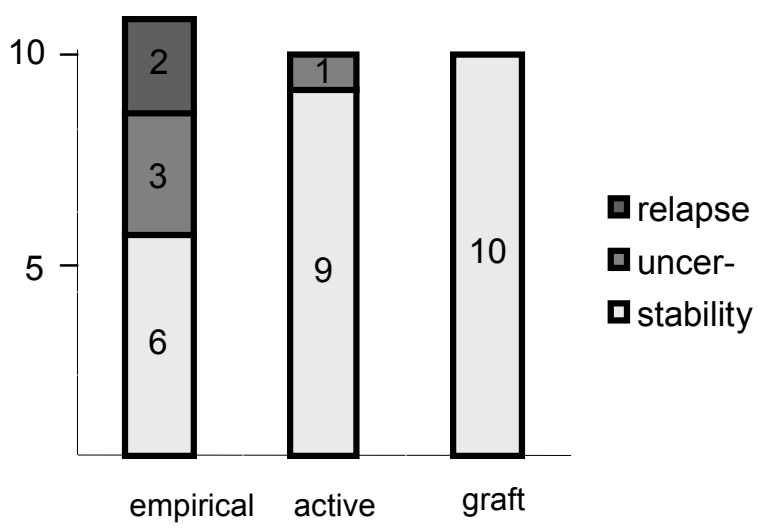

Figure 5: Stability of mandibular position

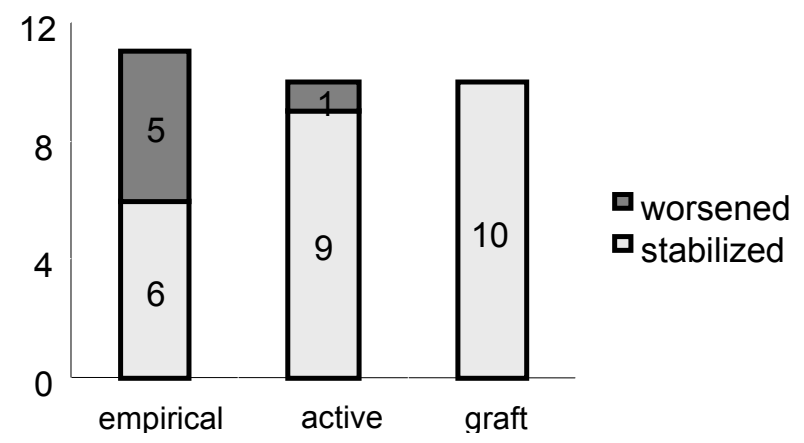

Figure 6: Occurrence of TMJ dysfunction

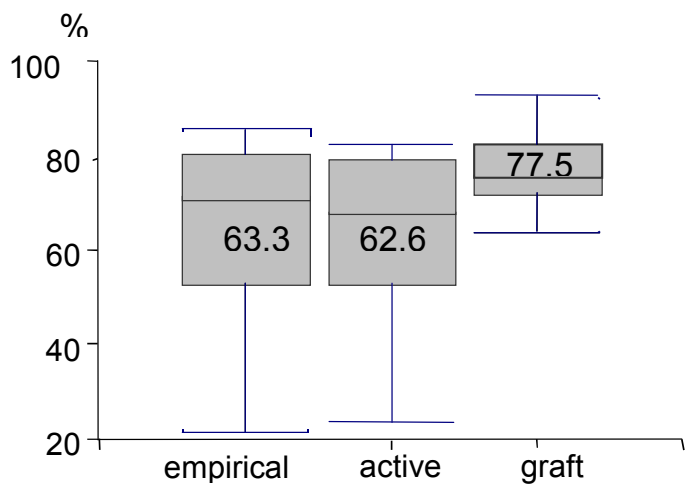

Figure 7: Recovery of mandibular movements at 6 months 


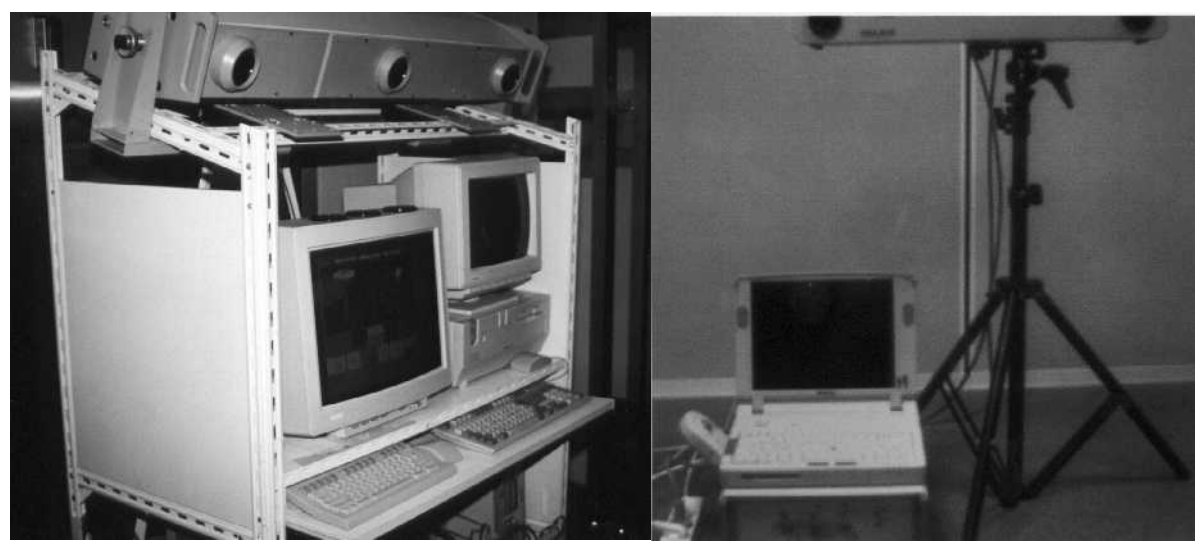

Figure 8: The different prototypes

- $\quad$ Figure 8A: the first version with its Unix station, and the Optotrak ${ }^{\mathrm{TM}}$ commanded by its dedicated PC;

- $\quad$ Figure 8B: the new platform with a single PC, a foot switch command and a smaller localizer (Polaris ${ }^{\mathrm{TM}}$ )

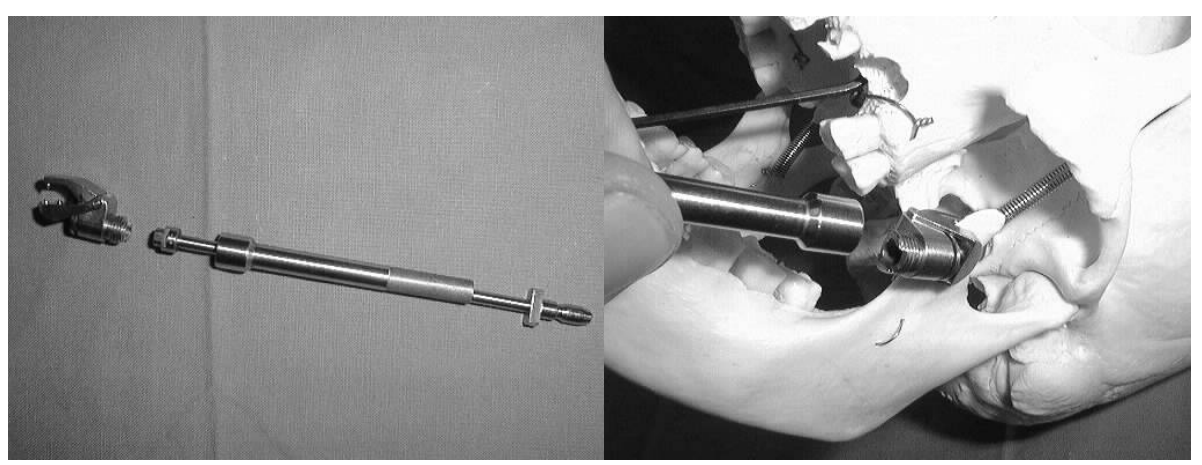

Figure 9: The new ancillary. A small vise is fixed on the coronoid process of the mandible. This vise hold the intermediate stem on which is fixed the rigid body 